\title{
Resonance Micro-Weighing of Sub-Picogram Mass with the Use of Adaptive Interferometer
}

\author{
Roman Romashko ${ }^{1,2}$, Timofey Efimov ${ }^{1}$, Yuri Kulchin ${ }^{1,2}$ \\ ${ }^{1}$ Institute of Automation and Control Processes FEB RAS, Lab. of Precision Optical Measurement Techniques, Radio st. 5, \\ 690041 Vladivostok, Russia, romashko@iacp.dvo.ru \\ ${ }^{2}$ School of Natural Sciences, Far-Eastern Federal University, Suhanova st. 8, 690091 Vladivostok, Russia, \\ tim2vl@yandex.ru
}

\begin{abstract}
Mass of macroscopic object is easily measured by a suitable balance. However, this approach becomes inapplicable if mass of microscopic object is to be determined. Alternative approach for mass measurement is based on using the micromechanical resonator as an inertial balance where oscillation frequency is shifted by small quantities of adsorbed mass. In this work we present experimental results of applying an adaptive interferometry technique based on dynamic hologram recorded in photorefractive CdTe crystal for measuring picogram mass adsorbed on micromechanical resonators with dimensions $215 \times 40 \times 15$ $\mu^{3}$. It is also shown that the resonance micro-weighing system based on adaptive interferometer has potential for reducing the threshold of mass detection down to $10^{-17} \mathrm{~g}$ in the case of using a resonator with sub-micron dimensions.
\end{abstract}

Keywords: Mass measurement, resonance micro-weighing, adaptive interferometer, photorefractive crystal.

\section{INTRODUCTION}

$\mathrm{M}$ ASS OF macroscopic object is easily measured by a suitable balance [1]. However, this approach becomes inapplicable if mass of microscopic object (molecule, atom, bacterium, virus, etc.) is to be determined. Alternative approach for mass measurement is based on using the micromechanical resonator as an inertial balance where oscillation frequency is shifted by small quantities of adsorbed mass [1-5]. In such applications, an interferometer is considered as a promising tool for detecting the resonator oscillations which are of ultra-small amplitude [1]. However, due to its high sensitivity, the classical interferometer is open to an influence of external impacts such as temperature or air pressure fluctuations, industrial noise, etc., which worsen the accuracy and increase the mass detection threshold. Moreover, classical homodyne interferometer is very sensitive to the quality of probe beam. The last issue makes difficult or even impossible practical use of interferometers in those applications where submicron resonators that drastically disturb wave front of the probe beam are used. Alternatively an interferometer can be implemented with use of dynamic hologram (DH) recorded in a photorefractive crystal (PRC). Due to adaptive properties of DH such an interferometer can stably operate in industrial environment and with light waves having complicated wavefronts $[6,7]$.

In this work we present experimental results of applying an adaptive interferometry technique based on dynamic hologram recorded in photorefractive CdTe crystal for measuring picogram mass adsorbed on micromechanical resonators. It is shown that the resonance micro-weighing (RMW) system based on adaptive interferometer has a potential for reducing the threshold of mass detection down to $10^{-17} \mathrm{~g}$ in the case of using a resonator with sub-micron dimensions.

\section{EXPERIMENTAL SETUP}

The scheme of the RMW measurement system is shown in Fig.1. Silicon micro-cantilever which plays the role of the sensing element is mounted on the positioning system. In experiments, we used two cantilevers: cantilever A with dimensions $233 \times 45 \times 4 \mu^{3}$ and cantilever $B$ with dimensions $215 \times 40 \times 15 \mu^{3}$. Laser light pulses at wavelength $532 \mathrm{~nm}$ each of energy $0.5 \mathrm{~mJ}$ and duration $7 \mathrm{~ns}$ were used to excite the cantilever out-of-plane mechanical vibrations. These vibrations were measured by the adaptive interferometer which was implemented with the use of semiinsulating photorefractive CdTe crystal and CW light at wavelength $1064 \mathrm{~nm}$ generated by $\mathrm{Nd}: Y A G$ laser. The dynamic hologram was recorded in the crystal in the orthogonal 3D geometry of photorefractive victoral threewave mixing $[8,9]$ as shown in Fig.2. The diameters of the reference beam and the object beam in the crystal were $900 \mu \mathrm{m}$ and $68 \mu \mathrm{m}$ (in focal plane), respectively. Here the object wave being reflected from the cantilever propagates through the PRC along its principal axis [001] where it is mixed with two reference waves which propagate in mutually orthogonal directions along crystal axes [010] and [100]. The object beam is focused by a lens in a spot of diameter $36 \mu \mathrm{m}$ at the cantilever surface. Three-wave coupling in PRC provides polarization-insensitive phase-tointensity transformation in the object wave, while adaptive properties of DH automatically stabilize the working point of the interferometer.

The adaptive interferometer possesses a very high sensitivity to transient phase variations which corresponds to the detection threshold of $2.4 \times 10^{-8} \mathrm{rad}(\mathrm{W} / \mathrm{Hz})^{1 / 2}$. Thus, the interferometer which operates with the object beam of $1 \mathrm{~mW}$ power at wavelength $1064 \mathrm{~nm}$ is able to detect oscillations with the amplitude of $3 \AA$ in wide frequency band (up to $10 \mathrm{MHz}$ ). Moreover, a holographic principle of waves combining in photorefractive crystal ensures full matching of wave fronts of mixed light beams, no matter what distortions in the object beam appear. 


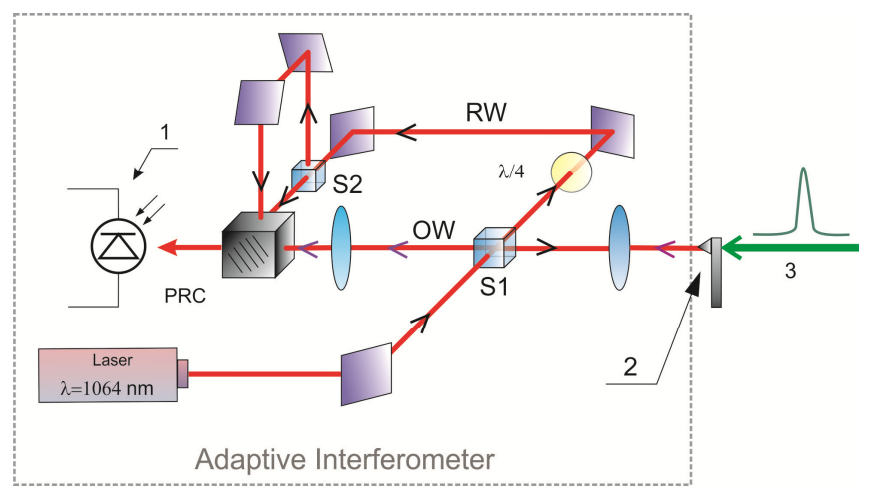

Fig.1. Scheme of the system for resonance micro-weighting based on adaptive interferometer: 1 - photodetector; 2 - cantilever; 3 laser pulse; RW - reference wave (which is split into two); OW object wave; S1, S2 - beam splitters; $\lambda / 4$ - quarter-wave plate.

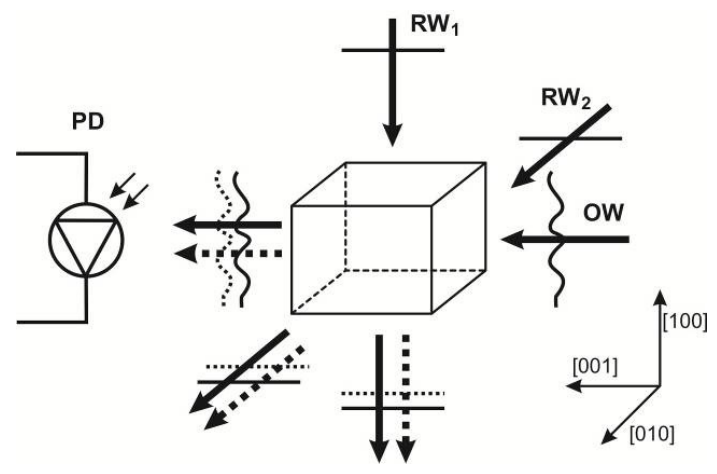

Fig.2. Dynamic hologram recording in the photorefractive crystal in 3D orthogonal geometry: $\mathrm{RW}_{1}$ and $\mathrm{RW}_{2}$ - reference waves; $\mathrm{OW}$ - object wave; PD - photodetector.

\section{EXPERIMENTAL RESULTS}

The waveform of the signal received from the photodetector after a laser shot which excites the cantilever is shown in Fig.3. The oscillations duration is about $0.5 \mathrm{~ms}$. Being received, the signal was recorded and processed for determining the oscillation frequency.

To demonstrate possibility of mass measurement the RMW system was tested with the use of cantilever A, which mass was varied. In the experiment, mass variation was done by change of the cantilever length. For this purpose the cantilever tip was exposed by accelerated Argon ions generated in the ion etching system HITACHI IM4000. The cantilever shapes before and after the cutting are shown in Fig.4. The length was reduced by $11 \mu \mathrm{m}$ (from $233 \mu \mathrm{m}$ down to $222 \mu \mathrm{m})$. Taking into account the shape of the cantilever tip which was slightly modified, the change of the mass was found to be equal to $5 \mathrm{ng}$.

In its turn, the Eigen frequency of the cantilever before and after cutting was experimentally found equal to $215.85 \mathrm{kHz}$ and $234.82 \mathrm{kHz}$, respectively. Calculation of mass change was done on the base of the model [4] which takes into account a localized mass attached to the cantilever. In numerical simulations, the length of the cantilever was calculated on the base of its oscillation frequency measured experimentally. The change in mass of the cantilever was found to be $4.2 \mathrm{ng}$.

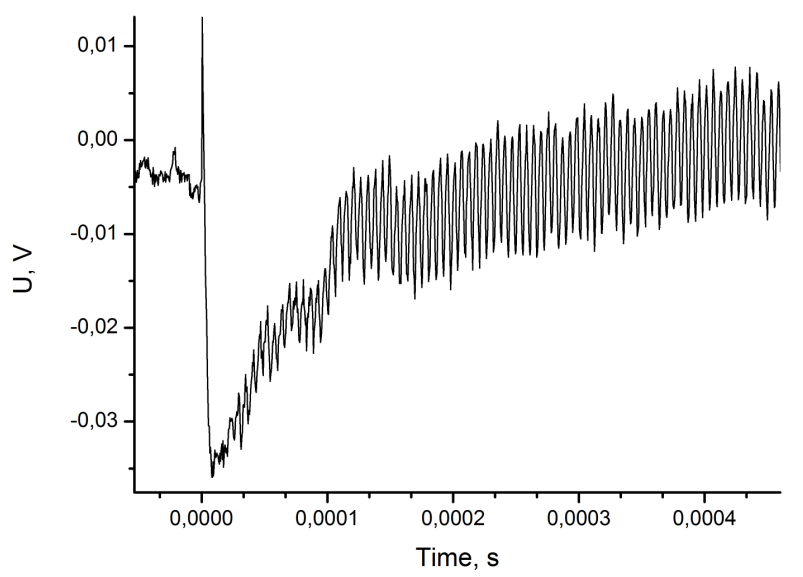

Fig.3. Waveform of the photodetector signal received after single laser shot exiting the cantilever (the laser pulse hits the cantilever at $t=0.0 \mathrm{~s})$.

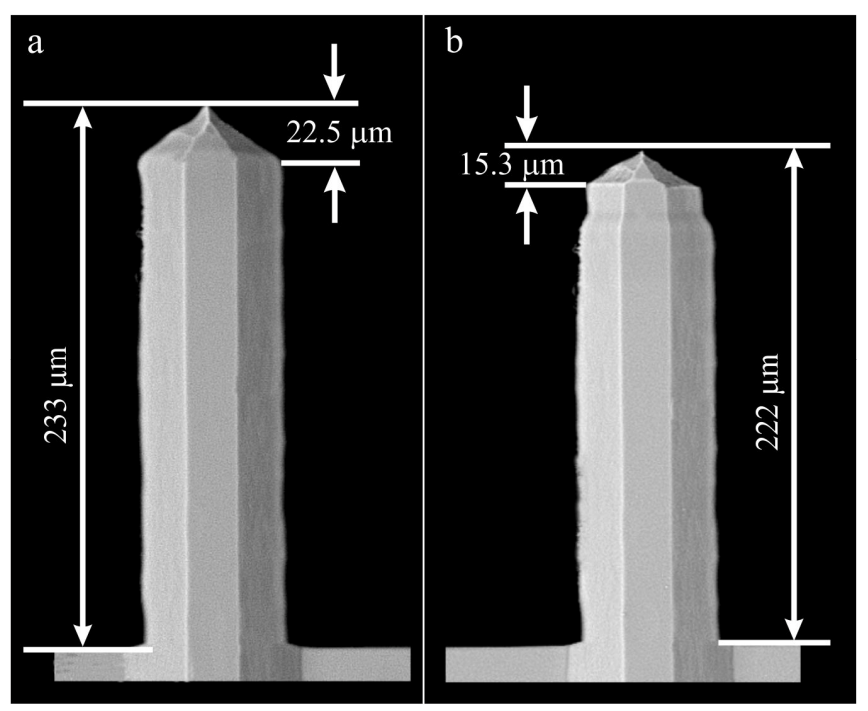

Fig.4. SEM images of the cantilever A: $a$ - original; $b$ - after cutting a tip by the ion etching system.

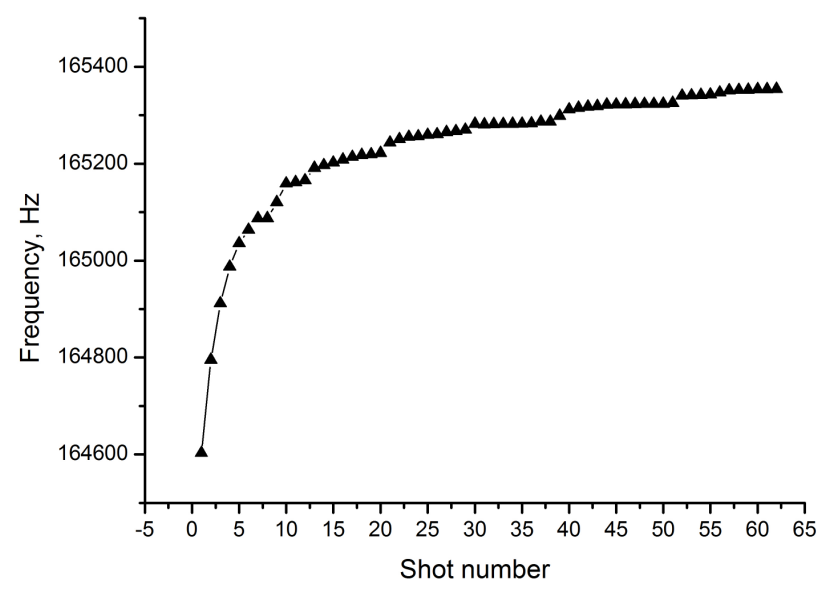

Fig.5. Resonance frequency of the cantilever vibration as function of laser shot number. 
The adaptive RMW system proposed in this work was applied for measuring mass of organic sub-microscopic objects with dimensions from 60 to $400 \mathrm{~nm}$ which were predeposited at the free end of the cantilever using the method of magnetron vacuum sputtering. In this experiment, cantilever B was used. In the process of measurement, each laser pulse exciting the cantilever caused detachment of certain number of nanoparticles from the cantilever which resulted in change of the cantilever resonant frequency. Fig.5. shows the evolution of the frequency with laser pulse shots. Increase of the resonance frequency indicates a decrease in the mass absorbed by the cantilever which was partially removed during shots.

Assuming that the adsorbed mass is mostly deposited at the end of the cantilever, the relation between resonance frequency shift, $\Delta f$, and change of mass, $\Delta m$, can be found as follows [4]:

$$
\Delta f=-\left(\frac{f_{0}}{2 m_{0}}\right) \Delta m
$$

where $f_{0}$ is the resonant frequency of the cantilever, and $m_{0}$ is its initial mass.

By using (1), one can easily estimate mass of material lost during hits of laser pulses. For experimental data shown in Fig.5., the change of mass was found equal to $420 \times 10^{-12} \mathrm{~g}$.

\section{THE THRESHOLD OF DETECTION OF THE MASS}

Resolution of mass measurement, $\delta m$, is determined by accuracy of a cantilever frequency measurement, $\delta f$. These parameters are related to each other as follows:

$$
\Delta m=\frac{m_{0}}{2} \frac{\delta f}{f_{0}}
$$

In our experiment the frequency was measured with accuracy $15 \mathrm{~Hz}$. Hence, the accuracy of the mass was $5.6 \times 10^{-12} \mathrm{~g}$.

The resolution $\delta m$ can also be considered as a minimal detectable mass or threshold of mass measurement. As seen from (2), in order to reduce the detection threshold it is necessary to reduce the total mass of the cantilever, $m_{0}$, what can be done by reducing its size. In this work, we studied the possibility of using ultra-small cantilevers in RMW system. For this purpose a micro-rod (used as an oscillator) was fabricated from a silica fiber by thermal pulling of its tip. The micro-rod has a cylindrical shape with a diameter of $300 \mathrm{~nm}$ (see inset in Fig.6.) and a length of 15 microns. The micro-rod was positioned in relation to the object beam of adaptive interferometer by means of XYZ micro-translator stage. Vibrations of the micro-rod were excited by electrodynamic transducer. The laser beam with focal point 34 microns illuminating micro-rod falls normally to its lateral surface.

Fig.6. shows the experimentally obtained dependence of the amplitude of the demodulation signal detected in the adaptive interferometer on the position of the object beam at micro-rod surface. As seen, the demodulation signal is absent until the distance from the center of the object beam to the end of the micro-rod is greater than the radius of the beam. The signal appears only when the object beam hits the micro-rod edge and achieves $12 \mathrm{~dB}$ SNR in the maximum when the distance is approximately equal to the beam radius. The subsequent sharp decrease in the signal, apparently, corresponds to the case when the object beam hits a junction of the micro-rod and the silica fiber, which leads to presumably lateral reflection of the object beam. Further increase in the signal amplitude occurs when the beam passes the junction area and is already reflected by the silica fiber.

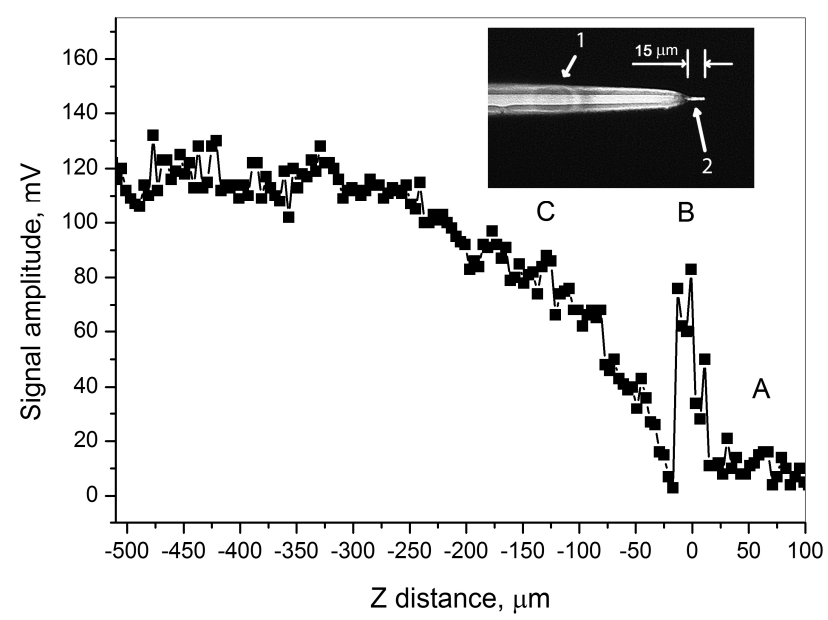

Fig.6. Demodulated signal amplitude as a function of object beam position in relation to the end micro-rod: 1 -silica fiber; 2 - microrod; A - object beam does not fall on micro-rod; B - object beam falls on micro-rod; $\mathrm{C}-$ object beam falls on silica fiber.

Obtained result experimentally confirms the possibility of using an oscillator with dimensions smaller than the wavelength of an object beam in a resonance microweighing system based on dynamic photorefractive hologram. It should be noted that light reflected from the micro-rod was very weak due to its cylindrical shape and low reflection of silica (reflecting coating was not applied to the micro-rod). The power of the reflected light was amounted to only $5 \mu \mathrm{W}$ which was significantly below the level of background radiation (caused by scattering of radiation in the crystal, optical elements, etc.) at a photodetector. Nevertheless, the measurement system based on adaptive interferometer was able to provide acceptable level of SNR. By assuming that the RMW system is built with the use of silicon cantilever with dimensions similar to those of tested micro-rod $\left(15 \times 0.3 \times 0.3 \mu \mathrm{m}^{3}\right)$, we can find by using (2) that such system can provide the threshold of mass detection equal to $5.8 \times 10^{-17} \mathrm{~g}$.

\section{CONCLUSIONS}

Preliminary results obtained allow one to conclude that resonance micro-weighing system based on adaptive holographic interferometer can be a promising tool for detection of ultra-small objects and measurement of their mass order of picogram. Adaptive holography principle 
opens the possibility of using ultra-small cantilevers with lower mass and higher Eigen frequency in such system and, as sequence, allow one to significantly reduce a threshold of mass detection down to tens of attogram.

\section{ACKNOWLEDGMENT}

The research is supported by Far-Eastern Federal University and Far Eastern Branch of Russian Academy of Sciences.

\section{REFERENCES}

[1] Wilkening, G., Koenders, L. (Eds.). (2005). Nanoscale Calibration Standards and Methods: Dimensional and Related Measurements in the Micro and Nanometer Range. John Wiley \& Sons.

[2] Carr, D.W., Evoy, S., Sekaric, L., Craighead, H.G., Parpia, J.M. (1999). Measurement of mechanical resonance and losses in nanometer scale silicon wires. Applied Physics Letters, 75 (7), 920-922.

[3] Gupta, S.V. (2008). Nano-technology for detection of small mass difference. MAPAN - Journal Metrology Society of India, 23 (3), 177-192.

[4] Ilic, B., Craighead, H.G., Krylov, S., Senaratne, W., Ober, C., Neuzil, P. (2004). Attogram detection using nanoelectromechanical oscillators. Journal of Applied Physics, 95 (7), 3694-3703.

[5] Singamaneni, S., LeMieux, M.C., Lang, H.P., Gerber, C., Lam, Y., Zauscher, S., Tsukruk, V.V. (2008). Bimaterial microcantilevers as a hybrid sensing platform. Advanced Materials, 20 (4), 653-680.

[6] Shandarov, S.M., Burimov, N.I., Kulchin, Y.N., Romashko, R.V., Tolstik, A.L., Shepelevich, V.V.E. (2008). Dynamic Denisyuk holograms in cubic photorefractive crystals. Quantum Electronics, 38 (11), 1059-1069.

[7] Kamshilin, A.A., Romashko, R.V., Kulchin, Y.N. (2009). Adaptive interferometry with photorefractive crystals. Journal of Applied Physics, 105 (3), 031101.

[8] Romasko, R.V., Kulchin, Y.N., Kamshilin, A.A. (2010). 3D-ortogonal three-wave mixing in adaptive interferometry. Pacific Science Review, 11 (5), 136138.

[9] Romashko, R.V., Kulchin, Y.N., Kamshilin, A.A. (2011). Polarization-Insensitive Adaptive interferometer based on orthogonal three-wave mixing in photorefractive crystal. Pacific Science Review, 13 (3), 252-254.

Received March 27, 2014. Accepted June 28, 2014. 